\title{
Diagnostic Value of Urinary MicroRNAs for Bladder Cancer in Asia
}

\author{
Yixin Cuil and Tian Zhou ${ }^{2}$ \\ ${ }^{1}$ Queen Mary School of Nanchang University, Nanchang University, Nanchang, Jiangxi, China \\ ${ }^{2}$ School of Basic Medical Sciences, Nanchang University, Nanchang, Jiangxi, China
}

\begin{abstract}
This study aimed to explore the diagnostic performance of urinary microRNAs (miRNAs) in bladder cancer (BC) in the Asian population. PubMed, Embase, Web of Science and Cochrane Library were searched for relevant literature on the diagnostic performance of miRNAs for BC in the Asian population. Subsequently, quality assessment on diagnostic accuracy studies-2 (QUADAS-2) was used for evaluating the quality of the included literature, and Stata version 15.0 for statistical analysis. Eleven published studies, including 1,220 BC patients, met the inclusion and exclusion criteria and, therefore, were investigated in this meta-analysis. The pooled sensitivity, specificity, positive likelihood ratio, and negative likelihood ratio of urinary miRNAs in the diagnosis of $\mathrm{BC}$ were 0.80 (95\% $\mathrm{Cl}$ : $0.74-0.85$ ), 0.76 (95\% Cl: $0.69-0.81), 3.28(95 \% \mathrm{Cl}: 2.63-4.10), 0.26(95 \% \mathrm{Cl}: 0.21-0.33)$, respectively. Additionally, the area under the curve (AUC) was 0.85 in the summary receiver operating characteristic (SROC) curve, and the diagnostic odds ratio (DOR) was 12.39 (95\%Cl: $9.00-17.07$ ). In conclusion, urinary miRNAs show good performance in diagnosing BC in Asia, and, therefore, can serve as effective biomarkers for early clinical screening and auxiliary diagnosis of BC.
\end{abstract}

Key Words: MicroRNAs, Bladder cancer, Diagnostic value, Meta-analysis.

How to cite this article: Cui Y, Zhou T. Diagnostic Value of Urinary MicroRNAs for Bladder Cancer in Asia. J Coll Physicians Surg Pak 2021; 31(10):1207-1213.

\section{INTRODUCTION}

Bladdercancer (BC) is a common malignancy of the urinary system, with an incidence ranking 11th among all malignancies and about 150,000 deaths per year. ${ }^{1}$ In China, about 78,100 new cases $(60,600$ males and 17,500 females $)$ of BC existed in 2014 , showing an incidence of $5.71 / 100,000(8.65 / 100,000$ in males and $2.62 / 100,000$ in females) and considered to be the most prevalent cancer involving the urinary tract. ${ }^{2}$ The rapid development of molecular biology technology has provided reliable detection indicators for the diagnosis of various diseases, and confirmed that the occurrence and development of malignant tumors are the result of multi-factor interaction. However, there is still a lack of effective markers to diagnose early BC.

MicroRNAs (miRNAs) are a class of highly conserved non-coding RNAs with a length of 18-25 nucleotides. In 1993, the first miRNA, miRNA-1in-4, was discovered in Caenorhabditis elegans, followed by the discovery of more than 700 human miRNAs in succession. ${ }^{3,4}$ With the deepening of research, scholars have found that miRNAs play a critical role in cell cycle regulation. Additionally, they can control cellular activities such as differentiation, growth, proliferation, and apoptosis by regulating target genes.

Correspondence to: Tian Zhou, School of Basic Medical Sciences, Nanchang University, Xuefu Avenue, Honggutan New District, Nanchang, Jiangxi 330031, China

E-mail:zhoutian@ncu.edu.cn

Received: April 07, 2021; Revised: July 19, 2021;

Accepted: September 08, 2021

DOI: https://doi.org/10.29271/jcpsp.2021.10.1207
According to the urological diseases guidelines, patients with painless hematuria should be thoroughly examined to detect potential urological cancers. ${ }^{7,8}$ Currently, white-light cystoscopy is considered a gold standard for the diagnosis and follow-up of $\mathrm{BC} .{ }^{9}$ However, this diagnostic approach is an invasive procedure resulting in pain and certain complications. In contrast with it, urine cytology has the advantages of non-invasive and high specificity, which has important clinical significance in detecting early $B C{ }^{10,11}$ Down-regulated miRNAs are considered tumor suppressor genes, while up-regulated ones as oncogenes. MiRNAs play dual roles in tumor inhibition and carcinogenesis in $B C$ cells, and can be found in the tumor tissue and in body fluids such as urine, blood, saliva, and peritoneal fluid. ${ }^{12,13}$ Urinalysis has significant advantages; firstly, the acquisition of urine is easier than that of blood and non-invasive; secondly, urine has direct contact with BC tissue, and, therefore, its miRNAs may better represent the disease process.

The value of urinary miRNAs in the diagnosis of $B C$ remains controversial in the Asian population. Therefore, the objective of this meta-analysis is to explore the diagnostic value of urinary miRNAs in BC in Asia, and to provide a medical evidence for the clinical diagnosis of early BC by urinary miRNAs.

\section{METHODOLOGY}

PubMed, Web of Science, Excerpta Medica Database (Embase), and Cochrane Library were searched upto March 2021, with an objective of collecting relevant English articles on the diagnostic value of urinary miRNAs for $B C$ in the Asian population. The literature retrieval strategy was as below: ("microRNA" or "miRNA") and 
("bladder cancer") and ("diagnosis"). In addition, related references of the included literature were searched manually to collect comprehensive data and information. The databases were searched by two researchers at the same time and cross-checked.

The inclusion criteria were patients in the case group who were were clinically diagnosed with BC; research subjects were Asians; and studies published in English language; and studied samples included urine. The exclusion criteria were overlapping publications; lack of data in literature; and low-quality literature.

The detailed data of the included studies were extracted and cross-checked by two investigators. Any differences produced by this process were resolved through discussion. The information extracted were first author, publication year, country, sample size, sampletype, miRNAs type, miRNAs detection method, sensitivity, and specificity. Quality assessment on diagnostic accuracy studies-2 (QUADAS-2) was applied to assess the quality of the included literature, with 11 items rated as Yes (1 point), Unclear ( 0 point), and No (-1 point). ${ }^{14}$ Finally, the total score was used as the evaluation standard for the quality of the included literature, with 0-6 classified as low-quality and 7-11 as high-quality.

Stata version 15.0 software was adopted to analyse the data. A bivariate mixed-effects model was applied to estimate the pooled sensitivity, specificity, diagnostic odds ratio (DOR), positive likelihood ratio (PLR), and negative likelihood ratio (NLR). Then, the summary receiver operating characteristic (SROC) curve was drawn and the area under the curve (AUC) was subsequently obtained and served to evaluate the diagnostic significance of urinary miRNA for BC detection. Finally, sensitivity analysis was conducted to verify the robustness of the findings. $P$ $<0.05$ was considered to be of statistical significance.

\section{RESULTS}

The detailed literature screening flowchart is displayed in Figure 1 , and the basic characteristics of the included studies are shown in Table I. Eleven articles were finally enrolled in this meta-analysis, including 1,220 cases and 974 controls. ${ }^{15-25}$ The Asian countries covered were China, South Korea, Japan and Iran. The urine samples were mainly urine supernatant, urine sediment and urine shedding. According to the quality assessment (Figure 2A-B), all the included literature was of high quality, all with scores of above 6 points.

Significant heterogeneity could be observed in sensitivity $\left(I^{2}=85.50 \%\right)$, specificity $\left(I^{2}=82.92 \%\right), \operatorname{PLR}\left(I^{2}=66.45 \%\right), \quad N L R$ $\left(I^{2}=78.42 \%\right)$ and DOR $\left(I^{2}=99.78 \%\right)$. The SROC curve (Figure 3$)$ was not in a typical shoulder-arm shape (Figure 2), with the spearman correlation coefficient of $0.407(P=0.105)$ between the logarithm of sensitivity and the logarithm of 1-specificity, indicating no significant threshold effect in this meta-analysis. The bivariate mixed-effects model results suggested that the pooled sensitivity was $0.80(95 \% \mathrm{Cl}: 0.74-0.85)$, specificity was $0.76(95 \% \mathrm{Cl}$ : $0.69-0.81)$, PLR was $3.28(95 \% \mathrm{Cl}: 2.63-4.10)$, NLR was 0.26 (95\% Cl: $0.21-0.33)$, DOR was 12.39 (95\%Cl: $9.00-17.07)$.

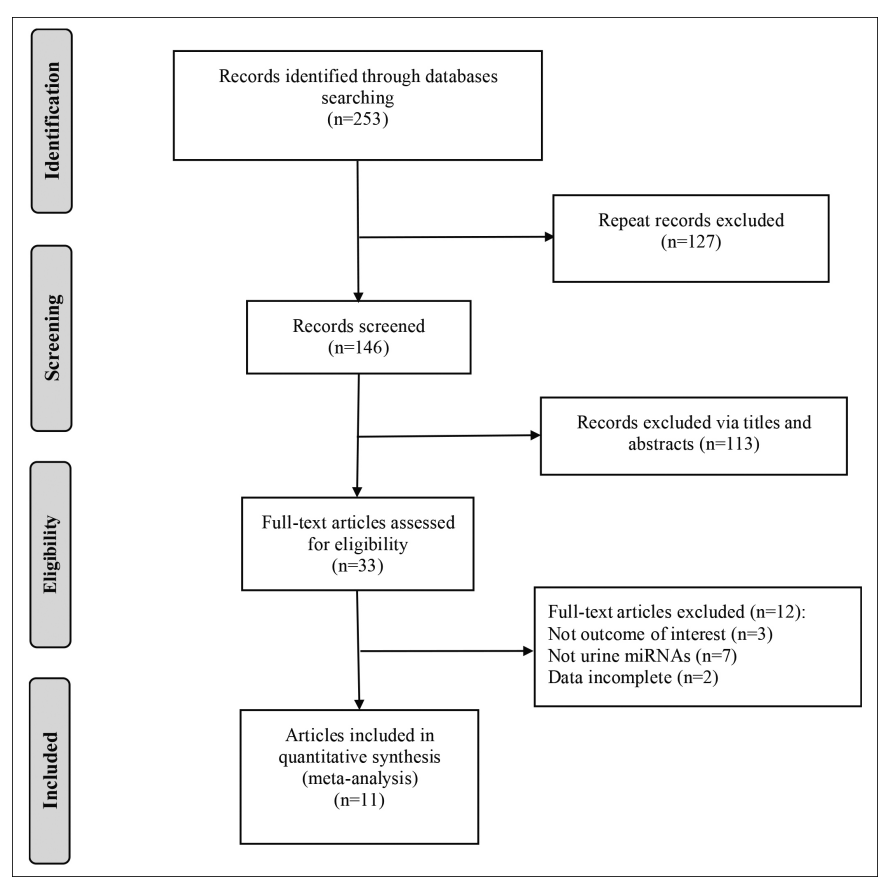

Figure 1: Flowchart of literature retrieval.

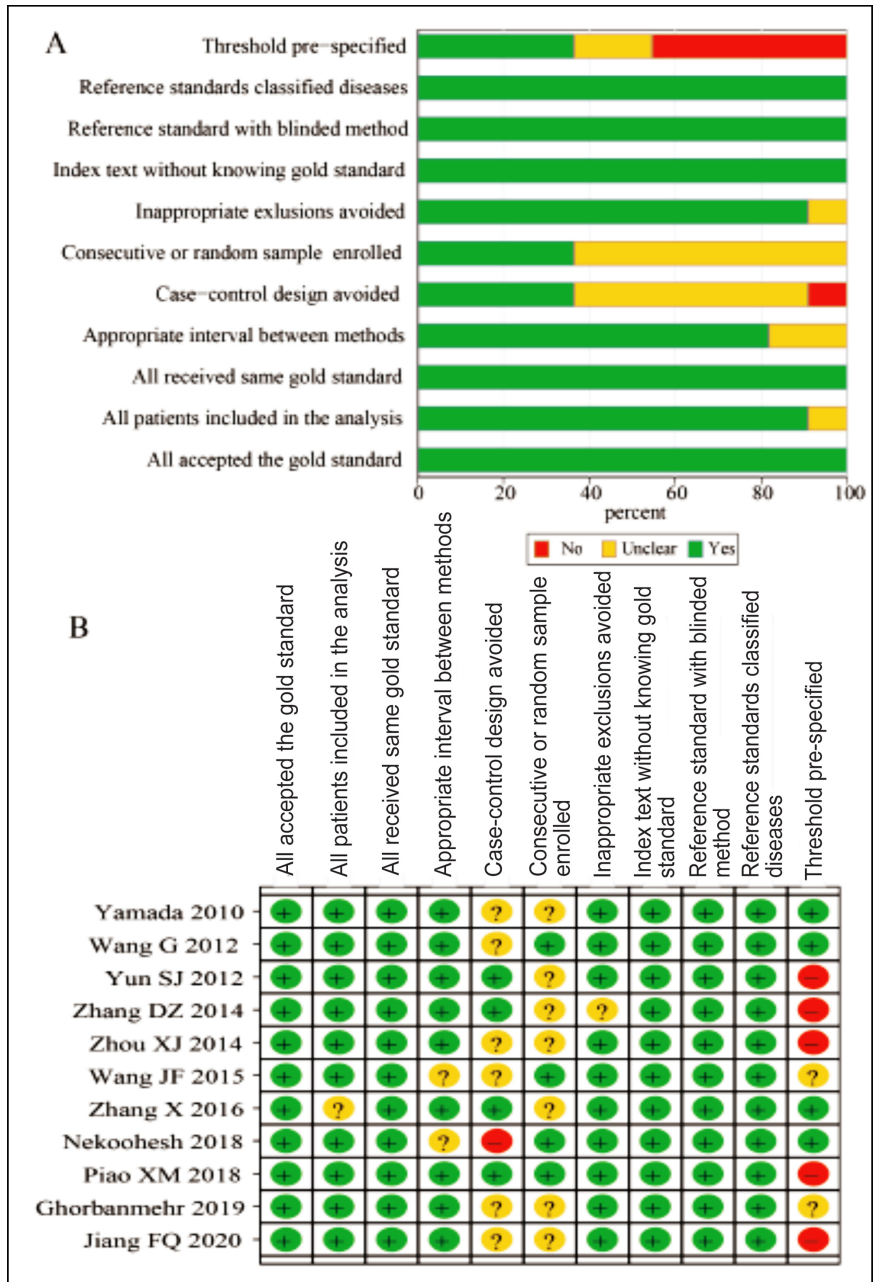

Figure 2: Quality assessment of included studies according to QUADAS-2 (A) Risk of bias graph; (B) Risk of bias summary). QUADAS: Quality assessment on diagnostic accuracy studies. 
Table I: Basic characteristics of the included studies.

\begin{tabular}{|c|c|c|c|c|c|c|c|c|c|c|}
\hline Study & Year & Country & Specimen & Method & miRNA profiling & Case & Control & $\begin{array}{c}\text { Sensitivity } \\
(\%)\end{array}$ & $\begin{array}{l}\text { Specificity } \\
(\%)\end{array}$ & QUADAS-2 \\
\hline \multirow{2}{*}{ Yamada $^{15}$} & \multirow{2}{*}{2010} & \multirow{2}{*}{ Japan } & Urine sediment & RT-PCT & miR-96 & \multirow{2}{*}{100} & \multirow{2}{*}{74} & 71 & 89.2 & \multirow{2}{*}{9} \\
\hline & & & & & miR-183 & & & 74 & 77.3 & \\
\hline Wang $\mathrm{G}^{16}$ & 2012 & China & Urine sediment & qRT-PCT & miR-200a & 51 & 24 & 100 & 52.6 & 10 \\
\hline Yun S $]^{17}$ & 2012 & Korea & Urine supernatant & RT-PCT & $\operatorname{miR}-145$ & 207 & 144 & 77.8 & 61.1 & 8 \\
\hline \multirow{3}{*}{ Zhang DZ ${ }^{18}$} & \multirow{3}{*}{2014} & \multirow{3}{*}{ China } & Urine supernatant & qRT-PCT & miR-99a and miR-125b & \multirow{3}{*}{50} & \multirow{3}{*}{21} & 90 & 90 & \multirow{3}{*}{7} \\
\hline & & & & & miR-99a & & & 78 & 85.7 & \\
\hline & & & & & miR-125b & & & 84.8 & 76.2 & \\
\hline Zhou XJ ${ }^{19}$ & 2014 & China & urine supernatant & qRT-PCT & miR-106b & 112 & 78 & 83 & 64.1 & 7 \\
\hline Wang $\mathrm{JF}^{20}$ & 2015 & China & urine supernatant & qRT-PCT & $\operatorname{miR}-214$ & 192 & 169 & 54.7 & 93.5 & 8 \\
\hline Zhang $X^{21}$ & 2016 & China & urine supernatant & qRT-PCT & miR-155 & 162 & 152 & 80.2 & 84.9 & 9 \\
\hline \multirow[b]{2}{*}{ Nekoohesh ${ }^{22}$} & \multirow[b]{2}{*}{2018} & \multirow[b]{2}{*}{ Iran } & Urine sediment & qRT-PCT & miR-34b and miR-10b & \multirow[b]{2}{*}{66} & \multirow[b]{2}{*}{53} & 59.1 & 78.8 & \multirow[b]{2}{*}{8} \\
\hline & & & & & $\begin{array}{l}\text { miR-141, miR-34b, } \\
\text { miR-10b and miR-103 }\end{array}$ & & & 75 & 63.5 & \\
\hline Piao $\mathrm{XM}^{23}$ & 2018 & Korea & urine supernatant & RT-PCT & miR-6124/miR-4511 & 117 & 97 & 91.5 & 74.2 & 9 \\
\hline \multirow{3}{*}{ Ghorbanmehr $^{24}$} & \multirow{3}{*}{2019} & \multirow{3}{*}{ Iran } & Urine sediment & qRT-PCT & miR-21-5p & \multirow{3}{*}{45} & \multirow{3}{*}{42} & 84 & 59 & \multirow{3}{*}{8} \\
\hline & & & & & miR-141-3p & & & 71 & 71 & \\
\hline & & & & & miR-205-5p & & & 82 & 62 & \\
\hline Jiang $\mathrm{FQ}^{25}$ & 2020 & China & Urine sediment & qRT-PCT & miR-192 & 118 & 120 & 78 & 76.7 & 7 \\
\hline
\end{tabular}

qRT-PCR: Quantitative real-time polymerase chain reaction; QUADAS: Quality assessment of diagnostic accuracy studies.

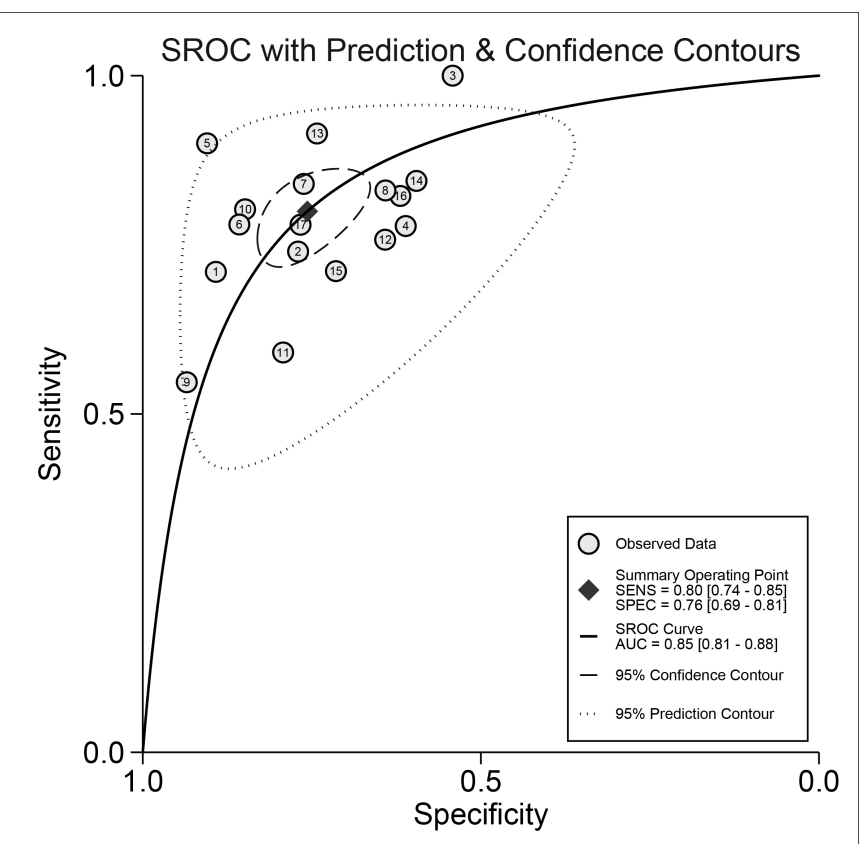

Figure 3: SROC curve of the diagnostic performance of urinary miRNAs in Asian patients with bladder cancer. SROC: summary receiver operating characteristic.

The forest plots of DOR, sensitivity and specificity, PLR and NLR are shown in Figure 4A-C, respectively. AUC was 0.85 (95\% Cl: 0.81-0.88). Fagan's nomogram indicated that, with the pre-test probability $20 \%$, the post-test probability of PLR was $45 \%$, while that of NLR was $6 \%$ (Figure 4D). This result suggested that urinary miRNAs had a good diagnostic performance for BC in the Asian population. The Deeks' funnel plot (Figure 5) showed a p-value of 0.57 , indicating no significant publication bias.

Further, subgroup analysis meta-regression was performed to figure out the source of heterogeneity. The results indicated that year of publication, the source of urine samples, miRNA type, and sample size might be the main sources of heterogeneity (Figure 6). Studies with a joint diagnosis of multiple miRNAs and a sample size more than 100 showed high diagnostic sensitivity.

The sensitivity analysis result is shown in Figure 7. The goodness-of-fit and bivariate normal distribution (Figure 7A-B) suggested that the bivariate mixed effects model for meta-analysis was robust. In addition, through influence analysis (Figure 7C) and outlier detection (Figure 7D), it was found that there were two studies that may have a greater impact on the results. ${ }^{16,20}$ After omitting the two, no significant change was found in sensitivity $(0.80$ vs. 0.79$)$, specificity (0.76 vs. 0.75$)$, PLR (3.28 vs. 3.10), NLR (0.26 vs. 0.28$)$, DOR (12.39 vs. 11.00), and AUC (0.85 vs. 0.84$)$ between the overall analysis with and without the outliers. This suggested that the conclusions obtained in this meta-study were robust. 


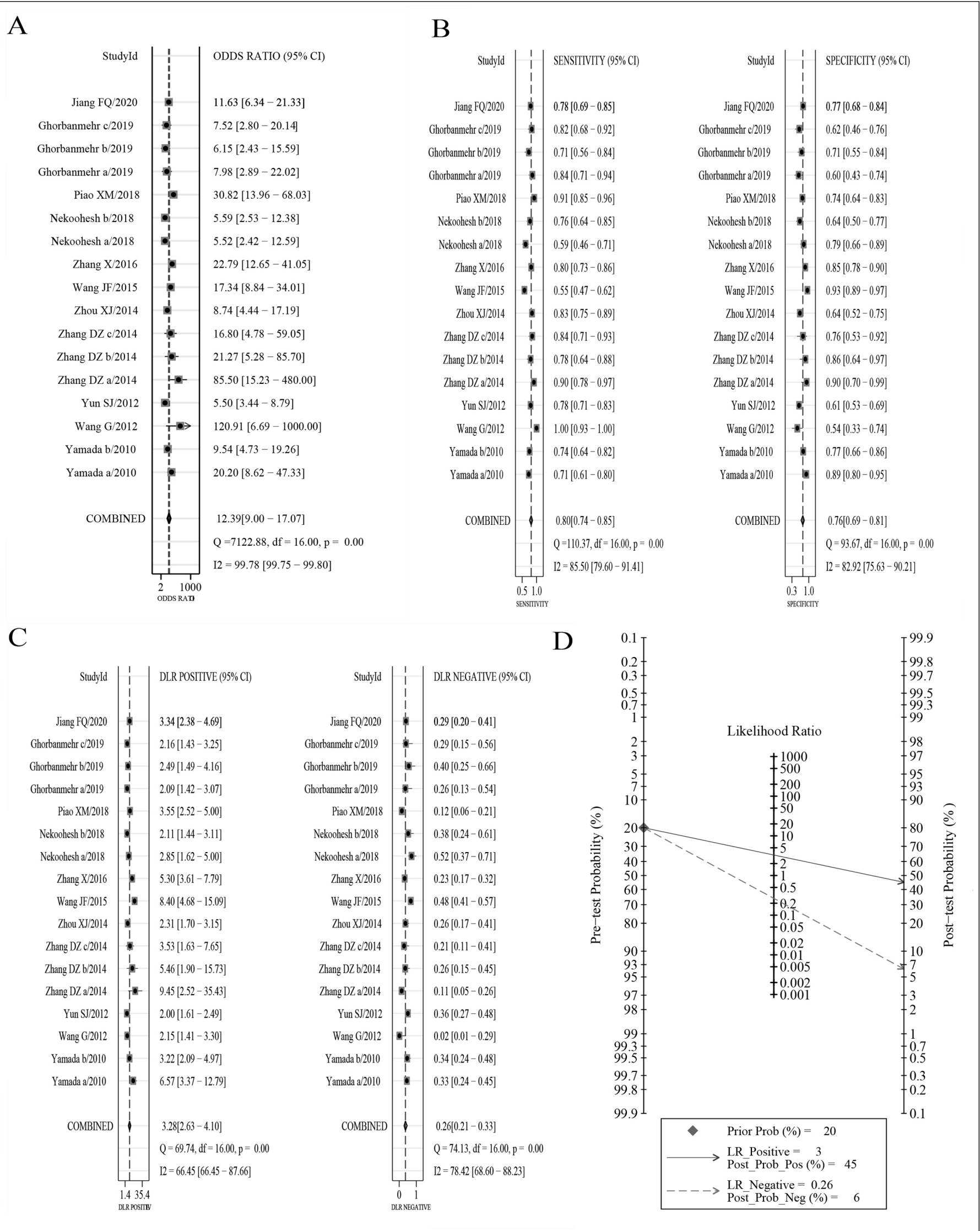

Figure 4: Diagnostic value of urinary miRNAs in Asian patients with bladder cancer (A) Diagnostic odds ratio; (B) sensitivity and specificity; (C) Positive likelihood ratio and negative likelihood ratio; (D) Fagan's nomogram). 


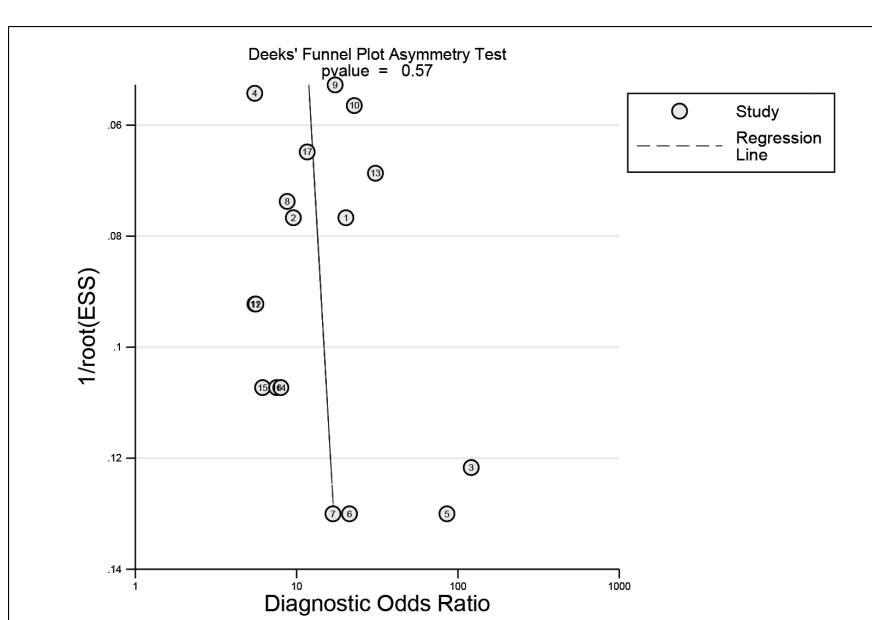

Figure 5: Deeks' funnel plot for assessing publication bias.

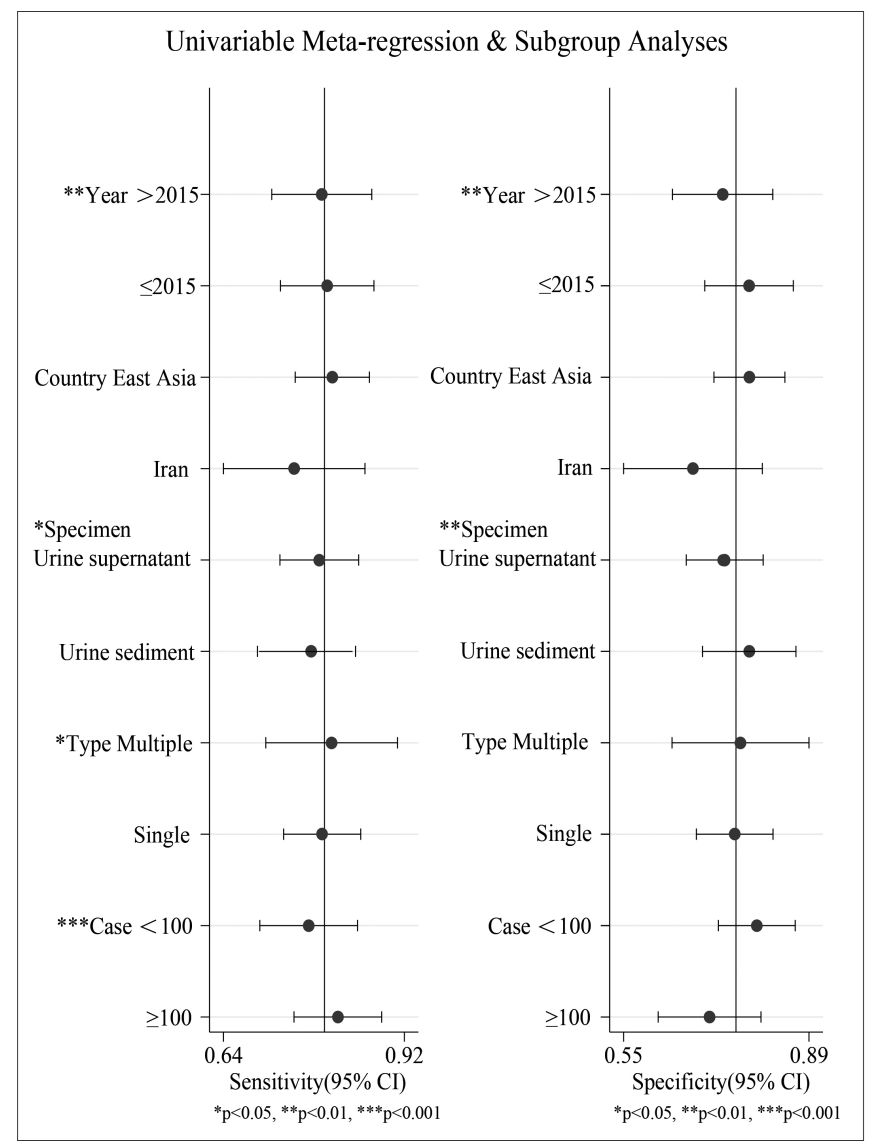

Figure 6: Univariable meta-regression and subgroup analyses.

\section{DISCUSSION}

This meta-analysis was carried out to explore the diagnostic value of urinary miRNAs for BC in the Asian population. This diagnostic approach, based on detection of human excreta, reduces pain due to blood collection and the risk of infectious diseases, which are promising for the early screening of BC. This kind of cancer is one of the most common malignant tumors in the population, especially in males. Currently, improving the diagnosis rate and treatment level and reducing the disease burden on patients and society are the common aims of both BC patients and clinicians. ${ }^{26}$ (a) Goodness-Of-Fit

(c) Influence Analysis

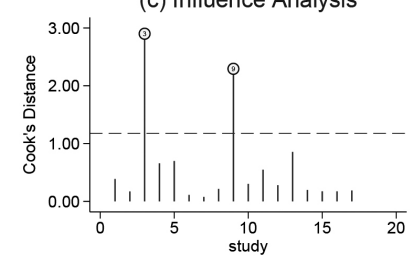

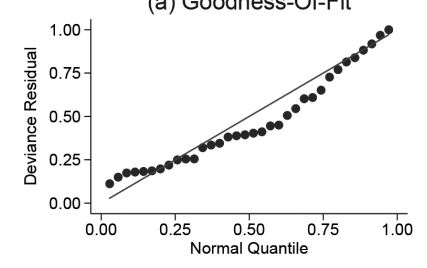

(b) Bivariate Normality
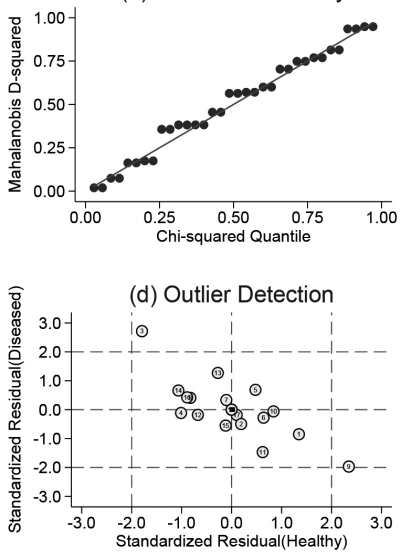

Figure 7: Sensitivity analysis to evaluate the robustness of the conclusions (a) Goodness-of-fit; (b) Bivariate normality; (c) Influence analysis; (d) Outlier detection).

The research results of miRNAs have provided new clues for the diagnosis and treatment of BC. MiRNAs are single-stranded non-coding small molecular fragments of RNA, and their physical properties are stable. Under extreme environments such as strong acid or alkali, high or low temperature, and even RNA digestive enzymes, the degree of degradation is low, so the integrity of miRNAs can be assured in the urine environment. ${ }^{27}$ MiRNAs can be used as biomarkers for a variety of diseases, especially for tumors, such as gastric cancer, liver cancer, lung cancer, breast cancer. ${ }^{28-31}$ Moreover, miRNAs are also of great value in tumor immunity; they are expected to become targets for drug treatment of diseases and to become one of the main approaches for the treatment of various tumors in the future. ${ }^{32,33}$

On the basis of strict inclusion and exclusion criteria, 11 studies were finally included in this meta-analysis. The results suggested that urinary microRNAs had good diagnostic performance for BC in Asian population, with AUC up to 0.85 . The sensitivity was as high as 0.80 , and the specificity was above 0.7 . The DOR is 12.39 , with the difference statistically significant, indicating that urinary miRNAs are valuable for the diagnosis of bladder cancer in Asian populations. The PLR and NLR were are 3.28 and 0.26 , respectively, which indicates that the urinary miRNAs in diagnosing BC in Asian populations were 3.28 times more likely to be true positives than false positives, and false negatives were $23 \%$ more likely to be true negatives. Generally, PLR $>10$ and NLR $<0.1$ suggest the possibility of diagnosing or excluding a disease is significantly increased. ${ }^{34}$ Fagan's nomogram results showed that at a pre-test probability of $20 \%$, the diagnostic accuracy after urine miRNAs testing increased to $45 \%$ if the result was positive, reduced to $6 \%$ if the result was negative. In practice, relying solely on urinary miRNAs to diagnose or exclude BC has certain limitations, and therefore a combination of it with $\mathrm{BC}$ clinical manifestations and other auxiliary examinations is introduced to make a correct diagnosis. From the results of publication bias, the funnel plot was basically symmetric, without statistically 
significant difference, indicating no significant publication bias in this study. In terms of sensitivity analysis, there are two studies that may have a certain impact on the results, but the diagnostic performance after excluding these two studies is not significantly different from the diagnostic performance before the elimination, indicating that the conclusions of this study are relatively robust.

The analysis results of heterogeneity showed that there was significant heterogeneity in sensitivity, specificity, DOR. The correlation coefficient between the logarithm of sensitivity and the logarithm of 1-specificity and SROC shape demonstrated that the heterogeneity were not from the threshold effect. Therefore, subgroup analysis and meta-regression analysis were subsequently conducted to explore the source of heterogeneity. The results suggest that year of publication, source of the urine samples, sample size and miRNA type have different effects on the heterogeneity. Joint diagnosis of multiple miRNAs and the sample size greater than 100 showed high diagnostic sensitivity. Therefore, in clinical practice, in order to improve the diagnostic performance of urinary miRNAs for BC in the Asian population, multiple miRNAs can be used for combined detection and diagnosis.

This study still has some limitations. First, different diagnostic criteria, such as the types and relative expression levels of miRNAs, may be a bias in the diagnostic results, and have a certain impact on the quality of the literature. Second, the included studies are relatively limited and the sample size is relatively small, which may affect the robustness of the results. Third, the Asian countries included in this study are limited as China, Japan, South Korea, and Iran; but lacking studies from other Asian countries, which may affect the applicability of the conclusions to the populations in other Asian countries. Fourth, this study only includes the literature on the diagnostic accuracy of urinary miRNAs in BC, but no studies with positive and negative effects on the results.

\section{CONCLUSION}

Urinary miRNAs have good diagnostic value on BC in Asian population, which is promising for the early diagnosis of $B C$. In future research, more attention should be paid to the combination of different miRNAs in the diagnosis of $B C$, so as to improve its diagnostic performance. Due to the limitations of this study, more clinical trials are required to verify the above conclusions before urinary miRNAs can be used in routine clinical practice.

\section{FUNDING:}

This work was supported by the grant from the National Natural Science Foundation of China (Grant No. 31860268).

\section{CONFLICT OF INTEREST:}

The authors declared no conflict of interest.

\section{AVAILABILITY OF DATA AND MATERIAL:}

The datasets used and/or analysed during the current study are available from the corresponding author on reasonable request.

\section{AUTHORS' CONTRIBUTION:}

YC, TZ: Critical revision of the manuscript, substantial contribution to the conception and design of the work, manuscript drafting, acquisition, analysis, and interpretation of the data, revising the manuscript critically, final approval of the version to be published.

Both the authors have read and approved the final manuscript.

\section{REFERENCES}

1. Siegel R, Miller K, Jemal A. Cancer statistics, 2019. Ca Cancer J Clin 2019; 69(1):7-34. doi: 10.3322/caac.21551.

2. He YT, Li DJ, Liang D, Zheng RS, Zhang SW, Zeng HM, et al. Incidence and mortality of bladder cancer in China. Chin J Oncol 2018; 40(9):647-52. doi: 10.3760/cma.j.issn.0253-3766. 2018.09.002.

3. Lee R, Feinbaum R, Ambros V. The C. Elegans heterochronic gene lin-4 encodes small RNAs with antisense complementarity to lin-14. Cell 2019; 75(5):843-54. doi: 10.1016/0092 $\mathrm{X}-8674(93) 90529$.

4. Yu W, Lee C. MicroRNA and cancer-focus on apoptosis. J Cell Mol Med 2009; 13(1):12-23. doi: 10.1111/j.1582-4934.2008. 00510.x.

5. Jee $\mathrm{YH}$, Wang J, Yue S, Jennings $M$, Clokie SJ, Nilsson O, et al. mir-374-5p, mir-379-5p, and mir-503-5p Regulate proliferation and hypertrophic differentiation of growth plate chondrocytes in male rats. Endocrinol 2018; 159(3):1469-78. doi: 10.1210/ en.2017-00780.

6. Li Q, Pan H, Liu Q. MicroRNA-15a modulates lens epithelial cells apoptosis and proliferation through targeting B-cell lymphoma-2 and E2F transcription factor 3 in age-related cataracts. Bioscience Rep 2019; 39(12). doi: 10.1042/BSR20191773.

7. Cha EK, Tirsar LA, Schwentner C, Christos PJ, Mian C, Hennenlotter J, et al. Immunocytology is a strong predictor of bladder cancer presence in patients with painless hematuria: a multicentre study. Eur Urol 2012; 61(1):185-92. doi: 10.1016/j.eururo.2011.08.073.

8. Kaufman DS, Shipley WU, Feldman AS. Bladder cancer. Lancet (London, England) 2009; 374(9685):239-49.

9. Chou R, Selph S, Buckley DI, Fu R, Griffin JC, Grusing S, et al. Comparative effectiveness of fluorescent versus white light cystoscopy for initial diagnosis or surveillance of bladder cancer on clinical outcomes: Systematic review and meta-analysis. J Urol 2017; 197(3 Pt 1):548-8. doi: 10.1016/j.juro.2016.10.061.

10. Junker K, Fritsch T, Hartmann A, Schulze W, Schubert J. Multicolor fluorescence in situ hybridisation (M-FISH) on cells from urine for the detection of bladder cancer. Cytogenet Genome Res 2006; 114(3-4):279-83. doi: 10.1159/000094214.

11. Grégoire M, Fradet $Y$, Meyer F, Têtu B, Bois R, Bédard G, et al. Diagnostic accuracy of urinary cytology, and deoxyribonucleic acid flow cytometry and cytology on bladder washings during followup for bladder tumors. J Urol 1997; 157(5):1660-64.

12. Lin $Y$, Chen $H, H u Z$, Mao $Y, X u X$, Zhu $Y$, et al. miR-26a inhibits proliferation and motility in bladder cancer by targeting HMGA1. Febs Lett 2013; 587(15):2467-73. doi: 10.1016/j.febslet.2013. 06.021 .

13. Ettore M, Giulia P, Giovanni C, Andrea B, Giulia EM, Stefano B. Expression of urinary miRNAs targeting NLRs inflammasomes in bladder cancer. Onco Ther 2017; 10:2665-73. doi: 10.2147/OTT.S132680.

14. Whiting P, Rutjes A, Westwood M, Mallett S, Deeks J, Reitsma J, 
et al. QUADAS-2: A revised tool for the quality assessment of diagnostic accuracy studies. Ann Intern Med 2019; 155(8):529-36. doi: 10.7326/0003-4819-155- 8-20111018000009.

15. Yamada Y, Enokida H, Kojima S, Kawakami K, Chiyomaru T, Tatarano S, et al. MiR-96 and miR-183 detection in urine serve as potential tumor markers of urothelial carcinoma: Correlation with stage and grade, and comparison with urinary cytology. Cancer Sci 2011; 102(3):522-29. doi: 10.1111/j.13497006.2010.01816.x.

16. Wang G, Chan S, Kwan C, Li K, Yip K, Szeto C, et al. Expression of microRNAs in the urine of patients with bladder cancer. Clin Genitourin Cancer 2012; 10(2):106-13. doi: 10.1016/j.clgc.2012.01.001.

17. Yun $S$, Jeong $P, \operatorname{Kim} W$, Kim $T$, Lee $Y$, Song $P$, et al. Cell-free microRNAs in urine as diagnostic and prognostic biomarkers of bladder cancer. Int J Oncol 2012; 41(5):1871-8. doi: 10.3892/ijo.2012.1622.

18. Zhang D, Lau K, Chan E, Wang G, Szeto C, Wong K, et al. CellFree Urinary MicroRNA-99a and MicroRNA-125b are diagnostic markers for the non-invasive screening of bladder cancer. Plos One 2014; 9(7):e100793. doi: 10.1371/journal.pone.0100793.

19. Zhou X, Zhang X, Yang Y, Li Z, Du L, Dong Z, et al. Urinary cellfree microRNA-106b as a novel biomarker for detection of bladder cancer. Med Oncol 2014; 31(10). doi: 10.1007/ s12032-014-0197-z.

20. Wang J, Zhang X, Wang L, Dong Z, Du L, Yang Y, et al. Downregulation of urinary cell-free microRNA-214 as a diagnostic and prognostic biomarker in bladder cancer. J Surg Oncol 2015; 111(8):992-99. doi: 10.1002/jso.23937.

21. Zhang $X$, Zhang $Y$, Liu X, Fang A, Wang C. Direct quantitative detection for cell-free miR-155 in urine: A potential role in diagnosis and prognosis for non-muscle invasive bladder cancer. Oncotarget 2015; 7(3):3255-66. doi: 10.18632/oncotarget.6487.

22. Nekoohesh L, Modarressi MH, Mowla SJ, Sadroddiny E, Etemadian $M$, Afsharpad $M$, et al. Expression profile of miRNAs in urine samples of bladder cancer patients. Biomark Med 2018; 12(12):1311-21. doi: 10.2217/bmm-2018-0190.

23. Piao XM, Jeong P, Kim YH, Byun YJ, Xu Y, Kang HW, et al. Urinary cell-free microRNA biomarker could discriminate bladder cancer from benign hematuria. Int J Cancer 2019; 144(2):380-88. doi: 10.1002/ijc.31849.

24. Gh Orbanmehr N, Gh Arbi S, Korsching E, Tavallaei M, Einollahi B, Mowla SJ. miR-21-5p, miR-141-3p, and miR-205-5p levels in urine-promising biomarkers for the identification of prostate and bladder cancer. Prostate 2019; 79(1):88-95. doi: 10.1002 /pros. 23714.

25. Jiang F, Li C, Han J, Wang L. Diagnostic value of combination of MicroRNA-192 in urinary sediment and B-Ultrasound for bladder cancer. Technol Can Res Treat 2020; 19. doi: 10.1177/ 1533033819894573.

26. Ebrahimi H, Amini E, Pishgar F, Moghaddam SS, Nabavizadeh B, Rostamabadi Y, et al. Global, Regional and National Burden of Bladder Cancer, 1990 to 2016: Results from the GBD Study 2016. J Urol 2019; 201(5):893-901. doi: 10.1097/JU. 0000000000000025.

27. Bracken CP, Scott HS, Goodall GJ. A network-biology perspective of microRNA function and dysfunction in cancer. Nat Rev Genet 2016; 17(12):719-32. doi: 10.1038/nrg.2016.134.

28. Lu X, Lu J, Wang S, Zhang Y, Ding Y, Shen X, et al. miR-92a-3p Circulating serum exosomal as a novel biomarker for early diagnosis of gastric cancer. Future Oncol 2021; 17(8):907-19. doi: 10.2217/fon-2020-0792.

29. Jiang $Y$, He J, Li Y, Guo Y, Tao $H$. The diagnostic value of MicroRNAs as a biomarker for hepatocellular carcinoma: A meta-analysis. Biomed Res Int 2019; 2019:5179048. doi: $10.1155 / 2019 / 5179048$.

30. Wu Y, Yin Q, Zhou YL, He L, Zou ZQ, Dai XY, et al. Evaluation of microRNAs as potential biomarkers in circulating HPV-DNA-positive non-small cell lung cancer patients. Cancer Biol Ther 2021; 22(2):136-48. doi: 10.1080/15384047.2021.1872155.

31. Luo ZB, Lai GE, Jiang T, Cao CL, Peng T, Liu FE. A competing endogenous RNA network reveals novel IncRNA, miRNA and mRNA biomarkers with diagnostic and prognostic value for early breast cancer. Technol Cancer Res Treat 2020; 19:1079250941. doi: $10.1177 / 1533033820983293$.

32. Li Y, He X, Zhang $X, X u Y$, Wu Y, Xu X. Immune-related microRNA signature for predicting prognosis and the immune microenvironment in hepatocellular carcinoma. Life Sci 2021; 265:118799. 016/j.lfs.2020.118799.

33. Tokumaru Y, Asaoka M, Oshi M, Katsuta E, Yan L, Narayanan S, et al. High expression of microRNA-143 is associated with favorable tumor immune microenvironment and better survival in estrogen receptor positive breast cancer. Int J Mol Sci 2020; 21(9). doi: 10.3390/ijms21093213.

34. Stengel D, Bauwens K, Sehouli J, Ekkernkamp A, Porzsolt F. A likelihood ratio approach to meta-analysis of diagnostic studies. J Med Screen 2003; 10(1):47-51. doi: 10.1258/0969141033 21610806. 